\title{
On the coherent inelastic binary and multiparticle processes in ultrarelativistic hadron-nucleus, photon-nucleus and nucleus-nucleus collisions
}

Valery V. LYUBOSHITZ*

Joint Institute for Nuclear Research (Dubna, Russia)

E-mail: Valery.Lyuboshitz@jinr.ru

\section{Vladimir L. Lyuboshitz}

Joint Institute for Nuclear Research (Dubna, Russia)

The coherent inelastic processes of the type $a \rightarrow b$, which may take place in the interaction of hadrons and $\gamma$ quanta with nuclei at very high energies ( the nucleus remains the same), are theoretically investigated. For taking into account the influence of matter inside the nucleus, the optical model based on the concept of refraction index is applied. Analytical formulas for the effective cross section $\sigma_{\mathrm{coh}}(a \rightarrow b)$ are obtained, taking into account that at ultrarelativistic energies the main contribution into $\sigma_{\mathrm{coh}}(a \rightarrow b)$ is provided by very small transferred momenta in the vicinity of the minimum longitudinal momentum transferred to the nucleus. It is shown that the cross section $\sigma_{\text {coh }}(a \rightarrow b)$ may be expressed through the "forward" amplitudes of inelastic scattering $f_{a+N \rightarrow b+N}(0)$ and elastic scattering $f_{a+N \rightarrow a+N}(0), f_{b+N \rightarrow b+N}(0)$ on a separate nucleon, and it depends on the ratios $L_{a} / R$ and $L_{b} / R$, where $L_{a}, L_{b}$ are the respective mean free paths in the nucleus matter for the particles $a, b$ and $R$ is the nuclear radius.

In doing so, several characteristic cases with different relations of the magnitudes $L_{a}, L_{b}, R$ are considered in detail. When $L_{a} / R \gg 1$, but $L_{b} / R \ll 1$ ( or, on the contrary, $L_{a} / R \ll 1$ but $L_{b} / R \gg 1$ ), then the cross section $\sigma_{\mathrm{coh}}(a \rightarrow b)$ is equal to the ratio of the "forward" cross sections of inelastic scattering $a+N \rightarrow b+N$ and elastic scattering of the particle $b$ (or, respectively, a) on a nucleon, multiplied by the cross section of scattering on the "black" nucleus $\pi R^{2}$. The cases $L_{a} / R \gg 1, L_{b} / R \lesssim 1$ and $L_{a} / R \gg 1, L_{b} / R \ll 1$ (for heavy nuclei) correspond, in particular, to the coherent production of vector mesons $\rho^{0}, \omega, \phi$ at the interaction of very high energy photons with nuclei, and some numerical estimates are made for the coherent process $\gamma \rightarrow \rho^{0}$ on the $P b$ nucleus. Meantime, when both the conditions $L_{a} / R \gg 1$ and $L_{b} / R \gg 1$ are satisfied, then $\sigma_{\text {coh }}(a \rightarrow b)$ is proportional to the factor $R^{4} / k^{2}$, where $k$ is the initial energy of the particle $a$ in the laboratory frame.

The formalism described above is generalized also for the case of coherent inelastic multiparticle processes on a nucleus of the type $a \rightarrow\left\{b_{1}, b_{2}, b_{3} \ldots . b_{i}\right\}$ and for the case of coherent processes in collisions of two ultrarelativistic nuclei.

XVII International Conference on Hadron Spectroscopy and Structure 25-29 September, 2017

University of Salamanca, Salamanca, Spain

${ }^{*}$ Speaker. 


\section{Momentum transfer at ultrarelativistic energies and coherent reactions on nuclei}

It is known that at ultrarelativistic energies the minimal longitudinal momentum, transferred to a nucleus, tends to zero and, in connection with this, the role of coherent processes increases.

Let $f_{a+N \rightarrow b+N}(\mathbf{q})$ be the average amplitude of an inelastic process $a+N \rightarrow b+N$ on a separate nucleon in the rest frame of the nucleus (laboratory frame). Here $\mathbf{q}=\mathbf{k}_{b}-\mathbf{k}_{a}$ is the momentum transferred to the nucleon, $\mathbf{k}_{a}$ and $\mathbf{k}_{b}$ are the momenta of the particles $a$ and $b$, respectively. In the framework of the impulse approximation [1], taking into account the interference phase shifts at the inelastic scattering of the particle $a$ on a system of nucleons, the expression for the effective cross-section of the coherent inelastic process $a \rightarrow b$ on a nucleus can be presented in the form:

$$
\sigma_{\mathrm{coh}}(a \rightarrow b)=\int\left|f_{a+N \rightarrow b+N}(\mathbf{q})\right|^{2} P(\mathbf{q}) d \Omega_{b} .
$$

where $d \Omega_{b}$ is the element of the solid angle of flight of the particle $b$ in the laboratory frame, and the magnitude $P(\mathbf{q})$ has the meaning of the probability of the event that at the collision with the particle $a$ all the nucleons will remain in the nucleus and the quantum state of the nucleus will not change:

$$
P(\mathbf{q})=\left|\int n(\vec{\rho}, z) \exp \left(-i \mathbf{q}_{\perp} \vec{\rho}\right) \exp \left(-i q_{\|} z\right) d^{2} \vec{\rho} d z\right|^{2} .
$$

Here $n(\mathbf{r})$ is the nucleon density normalized by the total number of nucleons in the nucleus $\left(\int n(\mathbf{r}) d^{3} \mathbf{r}=A\right)$; the axis $z$ is parallel to the initial momentum $\mathbf{k}_{a}, \mathbf{q}_{\perp}$ and $q_{\|}$are the transverse and longitudinal components of the transferred momentum, respectively.

It is easy to see that the momenta $|\mathbf{q}| \lesssim 1 / R$ (here $R$ is the nuclear radius), transferred to a nucleon, give the main contribution to the effective cross-section $\sigma_{\text {coh }}(a \rightarrow b)$ (1.1). In doing so, the recoil energy of the nucleus can be neglected, the effective flight angles for the ultrarelativistic particle $b$ are very small: $\theta \lesssim 1 / k R \ll 1$, where $k=E_{b} \approx E_{a}$. Then it is possible to assume in Eqs. (1.1) and (1.2) that:

$$
\left|\mathbf{q}_{\perp}\right|=k \theta, \quad q_{\|}=q_{\min }=\frac{m_{a}^{2}-m_{b}^{2}}{2 k},
$$

where $m_{a}$ and $m_{b}$ are the masses of the particles $a$ and $b$, respectively. Here $q_{\min }$ is the minimal transferred momentum corresponding to the "forward" direction.

In most cases the characteristic momentum transferred to the nucleus at the inelastic coherent scattering $(|\mathbf{q}| \sim 1 / R)$ is small as compared with the characteristic momentum transferred to the nucleon in the process $a+N \rightarrow b+N$. Thus, the amplitude $f_{a+N \rightarrow b+N}(\mathbf{q})$ in Eq. (1.1) can be replaced by its value $f_{a+N \rightarrow b+N}(0)$ corresponding to the flight of the particle $b$ in the "forward" direction. Taking into account that at small angles $\theta$ the solid angle in Eq. (1.1) is $d \Omega=\sin \theta d \theta d \phi \approx$ $d^{2} \mathbf{q}_{\perp} / k^{2}$ and using the properties of the two-dimensional $\delta$-function, we obtain - as a result of integration of Eq. (1.1) over the transverse transferred momenta and over the volume of the nucleus:

$$
\sigma_{\operatorname{coh}}(a \rightarrow b)=\frac{4 \pi^{2}}{k^{2}}\left|f_{a+N \rightarrow b+N}(0)\right|^{2} \int\left(\left|\int_{-\infty}^{\infty} n(\vec{\rho}, z) \exp \left(-i q_{\min } z\right) d z\right|^{2}\right) d^{2} \vec{\rho}
$$

where $q_{\min }$ is determined by Eq. (1.3). 
For a spherical nucleus with the radius $R$ and the constant density of nucleons $n_{0}=3 A / 4 \pi R^{3}$, Eq. (1.4) gives at sufficiently high energies, when $\left|q_{\min }\right| R \ll 1$ :

$$
\sigma_{\mathrm{coh}}(a \rightarrow b)=\frac{8 \pi^{3}}{k^{2}} n_{0}^{2}\left|f_{a+N \rightarrow b+N}(0)\right|^{2} R^{4}=\frac{9 \pi}{2 k^{2} R^{2}} A^{2}\left|f_{a+N \rightarrow b+N}(0)\right|^{2} .
$$

It should be noted that our consideration relates not only to binary reactions but also to multiparticle coherent processes $a \rightarrow b_{1}+b_{2}+\ldots b_{i}$ on nuclei at very high energies. In the general case, the vector $\mathbf{k}_{b}$ means the total momentum of the system $b=\left\{b_{1}, b_{2} \ldots b_{i}\right\}$ with the effective mass $m_{b}$, and the magnitude $\left|f_{a+N \rightarrow b+N}(0)\right|^{2}$ determines the cross section of the production of the system $b$, moving as a whole in the "forward" direction, at the collision of particle $a$ with the separate nucleon.

\section{Effect of matter inside the nucleus on coherent processes}

In the relations obtained above, the multiple scattering of the initial and final particles on nucleons of the nucleus was neglected. This is possible when the mean free paths of the particles $a$ and $b$ inside the nucleus are much greater than the nuclear radius $R$. Actually, the role of the nucleus matter may be essential - especially in the case of medium and heavy nuclei. For the analysis of the effects of matter inside the nucleus, we will apply the optical model of the nucleus at high energy based on the concept of the refraction index [1,2].

Taking into account the refraction indexes of the particles $a$ and $b$, the influence of the nucleus matter on the coherent inelastic processes implies the introduction of the additional complex phase shift into Eq. (1.4): the exponential factor $\exp \left(-i q_{\min } z\right)$ is replaced by $Q=\exp \left[-i q_{\min } z+\right.$ $i \delta(\vec{\rho}, z)]$. For a spherical nucleus with the constant density $n(\rho, z)=n_{0}$ inside the interval $0 \leq|z| \leq \sqrt{R^{2}-\rho^{2}}(\rho=|\vec{\rho}|)$ and $n(\rho, z)=0$ outside this interval, the additional phase inside the indicated interval is described by the equation:

$$
\delta(\rho, z)=\left(\chi_{a}-\chi_{b}\right) z+\left(\chi_{a}+\chi_{b}\right) \sqrt{R^{2}-\rho^{2}}, \quad 0 \leq|z| \leq \sqrt{R^{2}-\rho^{2}},
$$

where

$$
\chi_{a}=\frac{2 \pi n_{0}}{k} f_{a+N \rightarrow a+N}(0), \quad \chi_{b}=\frac{2 \pi n_{0}}{k} f_{b+N \rightarrow b+N}(0) .
$$

Here $f_{a+N \rightarrow a+N}(0)$ and $f_{b+N \rightarrow b+N}(0)$ are the average amplitudes of elastic scattering of the particles $a$ and $b$ on a nucleon at the zero angle in the laboratory frame. The relations (2.1) are valid at $\left|\chi_{a}\right| / k \ll 1,\left|\chi_{b}\right| / k \ll 1$.

After the replacement $q_{\min } z \rightarrow q_{\min } z-\delta(\vec{\rho}, z)$ in Eq. (1.4) and the integration over $z$, we obtain the following expression for the cross-section $\sigma_{\operatorname{coh}}(a \rightarrow b)$ :

$$
\begin{gathered}
\sigma_{\mathrm{coh}}(a \rightarrow b)=\frac{8 \pi^{3}}{k^{2}} n_{0}^{2} \frac{\left|f_{a+N \rightarrow b+N}(0)\right|^{2}}{\left|q_{\min }+\chi_{a}-\chi_{b}\right|^{2}} \times \\
\times \int_{0}^{R}\left|\exp \left[-2 i\left(q_{\min }-\chi_{a}\right) \sqrt{R^{2}-\rho^{2}}\right]-\exp \left[2 i \chi_{b} \sqrt{R^{2}-\rho^{2}}\right]\right|^{2} \rho d \rho .
\end{gathered}
$$




\section{Dependence of cross-sections of inelastic coherent processes on the nuclear radius}

Taking into account the optical theorem [3], we have: $\operatorname{Im} \chi_{a}=n_{0} \sigma_{a N} / 2, \operatorname{Im} \chi_{b}=n_{0} \sigma_{b N} / 2$, where $\sigma_{a N}$ and $\sigma_{b N}$ are the total cross-sections of interaction of the particles $a$ and $b$ with nucleons, averaged over the protons and neutrons of the nucleus.

Let us consider firstly the situation when the total cross-section of the interaction of the initial particle $a$ with nucleons is small, so that $\left|\chi_{a}\right| R \ll 1$, but $\left|\chi_{b}\right| R \gg 1$. In so doing, $L_{a} \gg R, L_{b} \ll R$, where $L_{a}=\left(n_{0} \sigma_{a N}\right)^{-1}$ and $L_{b}=\left(n_{0} \sigma_{b N}\right)^{-1}$ are the mean free paths of the particles $a$ and $b$, respectively, inside the nucleus. In particular, we can deal with the coherent production of vector mesons $\rho^{0}, \omega, \phi$ at the interaction of very high energy photons with heavy nuclei.

It follows from Eq. (2.2), neglecting the terms, depending on the masses $m_{a}$ and $m_{b}$ and tending to zero at very high energies, that:

$$
\sigma_{\mathrm{coh}}(a \rightarrow b)=\pi R^{2}\left|\frac{f_{a+N \rightarrow b+N}(0)}{f_{b+N \rightarrow b+N}(0)}\right|^{2} .
$$

Let us emphasize that, according to Eq. (3.1), the effective cross-section of the coherent process $a \rightarrow b$ on a nucleus at very high energies has the same dependence on the number of nucleons (proportional to $A^{2 / 3}$ ) as the cross-section of scattering of the final particle $b$ on the "black" nucleus, despite the smallness of the cross-section of interaction of the initial particle $a$ with a separate nucleon (in connection with this, see [4]).

For example, in the case of coherent process $\gamma \rightarrow \rho^{0}$ on the lead nucleus $\left(R=1.1 \cdot 10^{-13} A^{1 / 3}\right.$ $\left.\mathrm{cm} \approx 6.5 \mathrm{Fm}, L_{\rho} \sim 1.5 \mathrm{Fm},\left|f_{\gamma+N \rightarrow \rho+N}(0) / f_{\rho+N \rightarrow \rho+N}(0)\right|^{2} \sim 10^{-3}\right)$ the formula (3.1) is applicable at the energies of $\gamma$-quanta above several tens of $\mathrm{GeV}$ in the nucleus rest frame $\left(k \gg m_{\rho}^{2} L_{\rho} \sim\right.$ $4.5 \mathrm{GeV})$. In doing so, $\sigma_{\mathrm{coh}}\left(\gamma+P b \rightarrow \rho^{0}+P b\right) \sim 1.3 \mathrm{mbn}$.

On the other hand, it is easy to verify that, under the conditions $\left|q_{\min }\right| R \ll 1,\left|\chi_{a}\right| R \sim R / L_{a}$ $\ll 1,\left|\chi_{b}\right| R \sim R / L_{b} \ll 1$, the expansion of exponents in the expression (2.2) into the power series leads to the relation (1.5) - just as one would expect. In this limit, the cross-section $\sigma_{\text {coh }}(a \rightarrow b)$ is proportional to $R^{4}$ ( or to $A^{4 / 3}$ ).

For more details regarding the results of Sections 1 - 3, see, e.g., the paper [5] .

\section{The case of collisions of two ultrarelativistic nuclei}

At collisions of two ultrarelativistic nuclei, the coherent inelastic processes of the type $N \rightarrow$ $b=N+c(c=\pi, 2 \pi, \ldots)$ on the nucleus 2 , induced by the nucleons of nucleus 1 , and the analogous ones on the nucleus 1 , induced by the nucleons of nucleus 2 , may take place. In doing so, the nucleus being incident in fact (nucleus 1 in the first case and nucleus 2 in the second case ) is disintegrated and, thus, the contributions of constituent nucleons into the effective cross-section of the coherent process are summed up incoherently. This holds due to the following: if the coherent process takes place, e.g., on the nucleus 2, then in the rest frame of this nucleus the energy transfer to the nucleons at very high energies equals zero in fact, whereas the longitudinal transferred momentum amounts to :

$$
\left|q_{||}\right|=\left|q_{\min }\right|<<\frac{1}{R}
$$


where $R$ is the nuclear radius. Then, according to the Lorentz transformation, in the rest frame of the incident nucleus 1 we have :

$$
\left|\widetilde{q}_{||}\right|=\gamma\left|q_{\min }\right|=\frac{\left|m_{N}^{2}-m_{N+c}^{2}\right|}{m_{N}} .
$$

Here $\gamma$ is the Lorentz factor . In case of sufficiently large differences of the masses $m_{N}$ and $m_{N+c}$, the magnitude $\left|\widetilde{q}_{\|}\right|>>\frac{1}{R}$. Thus, we obtain :

$$
\sigma_{\mathrm{coh}}^{\left(A_{1}, A_{2}\right)}=A_{1} \sigma_{\mathrm{coh}}^{(2)}(N \rightarrow N+c)+A_{2} \sigma_{\mathrm{coh}}^{(1)}(N \rightarrow N+c),
$$

where $A_{1}$ and $A_{2}$ are respective numbers of nucleons in the nuclei 1 and 2, and $\sigma_{\operatorname{coh}}(N \rightarrow N+c)$ is determined in accordance with the general formula for the cross-section of the coherent inelastic process at the particle collision with a nucleus. Both the nuclei may remain unchanged, if the mass difference $\left|m_{N}-m_{N+c}\right|$ is very small - for example, in the case of production of electronpositron pairs at the collision of two ultrarelativistic nuclei $\left(c=e^{+} e^{-}\right)$.

\section{Summary}

In the present work ( see also, e.g., [5,6] ) the coherent processes at the interaction of ultrarelativistic particles with atomic nuclei are investigated. The role of these processes essentially increases at very high energies due to the fact that the minimal momentum, transferred to a nucleon, tends to zero with increasing energy. For the purpose of analyzing the influence of matter inside the nucleus on coherent reactions, the concept of refraction index is applied. The dependence of effective cross-sections of the coherent inelastic processes on the nuclear radius and the mean free paths of the initial and final particles in the nucleus matter is studied.

This consideration may be easily generalized also for the case of coherent inelastic multiparticle processes on a nucleus of the type $a \rightarrow\left\{b_{1}, b_{2}, b_{3} \ldots b_{i}\right\}$, as well as for the case of coherent processes at collisions of two ultrarelativistic nuclei.

\section{References}

[1]M. Goldberger, K. Watson, Collision theory ( New York - London - Sydney, 1964 ), Ch. 11.

[2]R. Jastrov, Phys. Rev. 82, 261 (1951) .

[3]L.D. Landau, E.M. Lifshitz, Quantum Mechanics. Nonrelativistic theory ( Nauka, Moscow, 1989 ), $\S 125$ [ transl. Pergamon Press, New York, 1977].

[4]V.N. Gribov, Zh. Eksp. Teor. Fiz. 57, 1307 (1969).

[5]V.L. Lyuboshitz, V.V. Lyuboshitz, Yad. Fiz. 70, 1224 (2007) [ Phys. At. Nucl. 70, 1185 (2007)] .

[6]V.V. Lyuboshitz and V.L. Lyuboshitz, in Proceedings of the ICHEP 2014 Conference ( Valencia, Spain, July 2 - 9, 2014 ), Nuclear and Particle Physics Proceedings, v. 273 - 275, 2758 (2016) . 